\title{
Inflamm-aging: The missing link to COVID-19 age-related mortality?
}

\author{
Nouran Eshak MD, ${ }^{1}$ Mahmoud Abdelnabi MD, ${ }^{2}$ Asmaa Beltagy MD ${ }^{3}$
}

The world is currently facing a COVID-19 pandemic; there are higher reported mortality rates in elderly patients than in children and younger adults. Caramelo et al. found that age was the strongest risk factor for COVID-19 related mortality and that patients 60 years or older have the highest risk. ${ }^{1}$ Riou et al. calculated age-specific case fatality ratios adjusted for asymptomatic cases and delayed mortality and found that these ratios escalated rapidly over 60 years of age and almost doubled every decade, reaching $18 \%$ in people more than 80 years. ${ }^{2}$

The higher mortality rates found in elderly patients have been attributed to immune suppression and the presence of co-morbidities, such as diabetes mellitus, cardiovascular diseases, lung diseases, obesity, etc. However, one of the core pathogenetic mechanisms of COVID-19 related tissue damage is the cytokine release syndrome or cytokine storm, which is characterized by a hyperactive innate immune response with the release of pro-inflammatory cytokines. Conti et al. found that higher levels of interleukin-1 (IL-1) and IL-6 were associated with more severe disease. ${ }^{3}$ Huang et al. reported that pro-inflammatory cytokines, including IL2, IL7, monocyte chemoattractant protein-1 (MCP-1), and TNF $\alpha$, were higher in COVID-19 patients who required ICU admission. ${ }^{4}$ Qin et al. also reported that significantly elevated IL2R, IL6, IL-10, and TNF $\alpha$ levels developed in more severe cases of COVID-19. ${ }^{5}$

An overall decline in the immune system with an inability to mount an effective immune response, "immunosenescence," and activation of the innate immune system with a resulting pro-inflammatory state called "inflamm-aging" occur with aging. With

Corresponding author: Nouran Eshak

Contact Information: Nouran.eshak@ttuhsc.edu DOI: 10.12746/swrccc.v8i34.701 advancing age there is an increase in the body's proinflammatory state, and older adults have higher levels of pro-inflammatory cytokines, such as IL-6 and TNF $\alpha$, and higher levels of acute phase reactants, such as C-reactive protein, than younger adults. Studies have shown that the process of inflamm-aging is associated with several diseases, including cardiovascular disease, insulin resistance, type 2 diabetes, and cancer. ${ }^{6}$ Several mechanisms may contribute to inflamm-aging, and these include oxidative stress, decreased autophagy, alteration in Toll-like receptor expression, and elevated levels of pro-inflammatory cytokines in the circulation, including IL-1, IL-6, TNF- $\alpha$, and PGE2. ${ }^{7}$ Some studies have shown that an increase in serum IL- 6 in the elderly is related to diseases, disability, and mortality. ${ }^{7}$ Therefore, the process of inflamm-aging with consequent cytokine storm rather than immunosuppression or immunosenescence may be responsible for the phenomenon of COVID-19 age-related mortality and disease severity seen in the elderly.

There are several preventive and therapeutic strategies that target inflamm-aging and could potentially ameliorate the more severe manifestations of COVID-19 seen in the elderly. Examples of possible therapies include some simple, widely available drugs and supplements, such as metformin, colchicine, hydroxychloroquine, and zinc. These drugs have modulated cytokine production in several animal and human studies. ${ }^{7,8}$ Zinc, a microelement, has interaction with the genes coding for IL-6, TNF alpha, and heat shock protein and has an essential role in regulating immunity and inflammation. Older adults are often zinc deficient, and adequate zinc supplementation has been associated with more healthy ageing and lower rates of infection. ${ }^{9}$ Zinc blocks coronavirus RNA polymerase activity in vitro and a zinc ionophore blocks virus replication in cell culture. ${ }^{10}$ Colchicine at a dose of $0.5 \mathrm{mg}$ daily led to a significantly lower risk of ischemic cardiovascular events, including death, than 
placebo in patients with a recent acute myocardial infarction. ${ }^{11}$ Several randomized controlled studies have begun to determine whether or not colchicine has any efficacy in patients with COVID-19. For example, The Greek Study in the Effects of Colchicine in Covid19 Complications Prevention (GRECCO-19) will try to determine if colchicine reduces myocardial injury and the development of pneumonia (NCT04326790). ${ }^{12}$ All these medications can be used potentially as preventive measures to limit hyperactivity of the immune system rather than treatment of immune-related damage that has already occurred.

Keywords: COVID 19, mortality, cytokine storm, inflamm-aging

From: ${ }^{1}$ The Department of Internal Medicine at Texas Tech University Health Sciences Center, Lubbock, Texas; ${ }^{2}$ Cardiology and Angiology Unit, Clinical and Experimental Internal Medicine Department, Medical Research Institute, Alexandria University, Alexandria, Egypt; ${ }^{3}$ Rheumatology Unit, Department of Internal Medicine, Faculty of Medicine, University of Alexandria, Egypt

Submitted: $4 / 18 / 2020$

This work is licensed under a Creative Commons Attribution-ShareAlike 4.0 International License.

\section{REFERENCES}

1. Caramelo F, Ferreira N, Oliveiros B. Estimation of risk factors for COVID-19 mortality-preliminary results. medRxiv 2020 .
2. Riou J, Hauser A, Counotte MJ, et al. Adjusted age-specific case fatality ratio during the COVID-19 epidemic in Hubei, China, January and February 2020. medRxiv. 2020.

3. Conti P, Ronconi G, Caraffa A, et al. Induction of pro-inflammatory cytokines (IL-1 and IL-6) and lung inflammation by COVID-19: anti-inflammatory strategies. J Biol Regulators Homeostatic Agents 2020;34(2).

4. Huang C, Wang Y, Li X, et al. Clinical features of patients infected with 2019 novel coronavirus in Wuhan, China. The Lancet 2020;395(10223):497-506.

5. Qin C, Zhou L, Hu Z, et al. Dysregulation of immune response in patients with COVID-19 in Wuhan, China. Clin Infect Dis 2020 Mar 12. pii: ciaa248. doi: 10.1093/cid/ciaa248.

6. Xia S, Zhang X, Zheng S, et al. An update on inflamm-aging: mechanisms, prevention, and treatment. J Immun Res 2016; 2016.

7. Bruunsgaard H, Andersen-Ranberg K, Hjelmborg J, et al. Elevated levels of tumor necrosis factor alpha and mortality in centenarians. Am J Med 2003;115(4):278-83.

8. Gao W, Xiong Y, Li Q, et al. Inhibition of toll-like receptor signaling as a promising therapy for inflammatory diseases: a journey from molecular to nano therapeutics. Frontiers Physiol 2017;8:508.

9. Mocchegiani E, Costarelli L, Giacconi R, et al. Nutrient-gene interaction in ageing and successful ageing. A single nutrient (zinc) and some target genes related to inflammatory/ immune response. Mechanisms Ageing Development 2006; 127(6):517-525.

10. Te Velthuis AJ, van den Worm SH, Sims AC, et al. Zn2+ inhibits coronavirus and arterivirus RNA polymerase activity in vitro and zinc ionophores block the replication of these viruses in cell culture. PLoS pathogens 2010;6(11).

11. Tardif JC, Kouz S, Waters DD. Efficacy and safety of lowdose colchicine after myocardial infarction. N Engl J Med 2019 Dec 26;381(26):2497-2505.

12. Deftereos SG, Siasos G, Giannopoulos G, et al. The GReek study in the Effects of Colchicine in COvid-19 complications prevention (GRECCO-19 study): rationale and study design. Hellenic J Cardiol 2020 Apr 3. 